CDU 616.314.18

Jorge Hugo Aydos*

*Prof. Adj. de Endodontia da Faculdade de Odontologia / UFRGS

R.Fac.Odont., Porto Alegre, 27:153-171, 1985. 


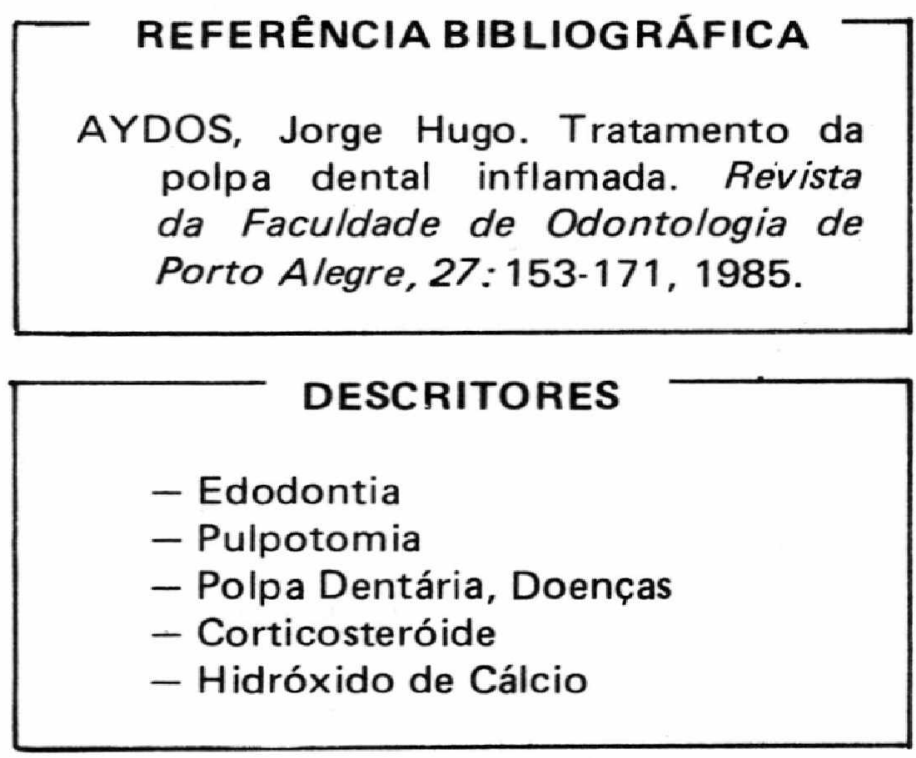

RESUMO
O autor avalia clínica e radiográ-
ficamente o comportamento da polpa
dental inflamada, submetida à pul-
potomia, ação de corticosteróide - an-
tibiótico e após, hidróxido de cálcio.
Como elemento auxiliar de diagnós-
tico e prognóstico é considerado, ex-
clusivamente, o "aspecto clinico visual
da polpa". O êxito foi de $94,3 \%$.




\section{TRATAMENTO DA POLPA DENTAL INFLAMADA}

\section{INTRODUÇÃo}

$O$ presente artigo se constitui num "resumo condensado", se assim se pode chamar, de um trabalho de pesquisa clínica e radiográfica no qual o objetivo maior foi a análise e a avaliação do comportamento da polpa dental inflamada quando submetida a tratamento conservador pela pulpotomia.

As vantagens na conservação da polpa são óbvias e não carecem defesa; o problema é saber-se a oprotunidade do tratamento conservador. "Salienta-se como uma das maiores dificuldades a determinação do estado pulpar previamente ao tratamento'.

Os resultados práticos da pulpotomia podem ser assim enumerados:

10 - Profilático: a permanência da polpa viva e sadia é certeza de saúde periapical.

20 - Biológico: Permite a complementação da rizogênese em dentes jovens, cujas polpas foram atingidas pela evolução da cárie, traumatismo, etc.

30. - Tećnico: tratamento mais rápido e tecnicamente mais fácil diante de dificuldades cirúrgicas intranponíveis, como morfologia endodôntica extremamente desfavorável.

40 - Econômico: a pulpotomia é, sabidamente, uma terapêtica mais rápida e menos dispendiosa do que o tratamento de canal. O fator econômico não pode ser desprezado, especialmente porque a nossa realidade revela que apenas $5 \%$ da população têm acesso ao tratamento endodôntico realizado por especialista em clínica particular. Além do que, sabe-se, os serviços socializados, assistenciais ou previdenciários que atendem a maioria da população, não oferecem tratamento endodôntico, e os casos de pulpites, notadamente em molares, são resolvidos, invarialmente, de maneira radical e mutilante pela exodontia.

Ao leitor interessado num estudo mais completo e detalhado sobre este tema, sugerimos a leitura do original deste trabalho, disponível na biblioteca da F.O.U.F.R.G.S. sob o título:

"Valor terapêutico da pulpotomia como opção no tratamento das pu/pites". (Investigação clinico-radiográfica em dentes humanos com polpas inflamadas submentidas à pulpotomia, aplicação de corticosteroides e, posteriormente, hidróxido de cálcio.

\section{REVISÃO DA LITERATURA}

A revisão da literatura, que por razões óbvias vai ser limitada à algumas poucas citações, registra de forma eloquente as mais discrepantes opiniões entre os autores, ficando evidente, desde logo, alguns pontos fundamentais de controvérsia e polêmica, a saber: diagnóstico pulpar e oportunidade do tratamento, técnica operatória, materiais e medicamentos empregados e resultados.

Relativamente ao diagnóstico pulpar, um número expressivo de autores $(9-11-16-17-18-22-27-28)$ comprovou experimentalmente a falta de correlação entre as manifestações clínicas e o quadro histopatológico da polpa. Outros, não obstante, (1 21 - $24-33$ ), pretendem através de sinais, sintomas e respostas aos testes auxiliares de diagnóstico (mecânicos, 
térmicos, e elétricos ), estabelecer o quadro histopatológico da inflamação pulpar. Outro grupo de autores (13 $14-15-29-31$ ), admite que as pulpites experimentalmente induzidas são lesões reversíveis. Certos autores, entretanto, (6 - 10 - 19-25 - 30 ), associam o termo "pulpite" com lesão irreversível e, consequentemente, prognóstico desfavorável.

Muitos pesquisadores (3 -8 - 23 32 ), mencionam a simultaneidade da lesão pulpar e periapical, e alguns consideram esta situação como definitivamente irreversível para a polpa.

Um fator de insucesso, muitas vezes, é representado por uma técnica inadequada. Alguns autores, por exemplo (20 - 26 - 34), utilizam a broca durante ou logo após a fase de remoção da polpa coronária. Este procedimento, como se sabe, é totalmente contra indicado.

Via (34), emprega antissépticos fortes e extremamente irritantes sobre a polpa, além de utilizar anestesia intra-pulpar.

No que respeita ao emprego de corticosteroides, Holland e colanoradores ( $13-14)$, verificaram que o processo inflamatório pulpar é um fenômeno reversível, tal como ocorre em tecido localizado em outras partes do organismo, desde que aplicada terapia adequada. Observaram, entretanto, que existe uma relação entre a intensidade da reação inflamatória e o resultado do tratamento. Assim, quanto mais intensa e extensa se apresenta a reação inflamatória, menor é a porcentagem de sucesso obtida após ao tratamento, apenas com hidróxido de cálcio. Entretanto, a aplicação de um curativo de corticosteroide associado a um antibiótico por 48 horas, antes da aplicação de hidróxido de cálcio, aumenta a porcentagem de sucesso em polpas severamente inflamadas, de $57 \%$ para praticamente $100 \%$.

Constataram, portanto, serem reais as possibilidades de recuperação da polpa dental, acometida de processo inflamatório, quando do emprego de corticosteroide-antibiótico.

Seltzer e colaboradores ( 29 ), afirmam que após o diagnóstico clínico e ao entrar na polpa, frequentemente somos surpreendidos pela descoberta de que o diagnóstico era incorreto. Isto é, a polpa apresenta um "aspecto clínico visual" que não comporta mais tratamento.

Filgueiras e colaboradores ( 7 ), sem citar especificamente o "exame clínico visual da polpa"' como elemento auxiliar de diagnóstico e prognóstico, dizem o seguinte: na realidade bom número de vezes verificamos, em pleno ato cirúrgico, que o diagnóstico e a indicação pré determinados não são os mais convenientes para aquele caso.

Isto devemos à precaridade dos recursos semiológicos de que dispomos.

Holland e Souza ( 11 ), demostraram histologicamente que pode ser obtida grande margem de sǔcesso no tratamento das polpas dentais inflamadasm enfatizando que no tratamento conservador, "'reveste-se de grande importância o exame clinico visual da polpa a ser tratada"'.

Bevilacqua ( 4 ), em sua Tese, afirma que na decisão do tratamento conservador da polpa dentária, o “diagnóstico anatômico" (aspecto clínico visual da polpa ) é de max́ima importância; e a dor apesar de seu valor semiológico inegável, pouco nos diz das condições daquele tecido. 


\section{PROPOSIÇÃo}

Partindo da rpremissa que pulpites experimentalmente induzidas são lesões reversíveis $(13-14-15-29-31)$, resolvemos investigar e avaliar clínica e radiograficamente, em dentes humanos submetidos à pulpotomia:

1 ㅇ - O potencial de recuperação da polpa dental quando inflamada pela evolução da cárie.

20. - Determinar até que ponto os diferentes graus de inflamação pulpar, estabelecidos pelo diagnóstico clínico como: pulpite infiltrativa incipiente, pulpite infiltrativa parcial, pulpite infiltrativa total, pulpite abscedada, pulpite ulcerada e pólipo pulpar, com ou sem lesão apical, se constituem em lesões reversíveis, quando submetidas à pulpotomia, ação de corticosteroides associados a antibiótico ou furacin e, posteriormente, hidróxido de cálcio.

30. - Avaliar o "exame clinico visual da polpa inflamada" como elemento auxiliar de diagnóstico e prognóstico das condições pulpares.

40 - Avaliar a ação de alguns corticosteroides associaddos a antibiótico ou furacin, como elementos coadjuvantes no tratamento conservador da polpa dental inflamada.

50 - Determinar a incidência de reabsorção dentinária interna após pulpotomia e aplicação de hidróxido de cálcio.

\section{MATERIAIS E MÉTODOS}

Os pacientes atendidos eram de ambos os sexos e de uma faixa etária variável de 12 a 40 anos. Foram selecionados dois grupos de dentes: molares superiores e inferiores.
Escolhido o caso, o paciente era submetido a exame clínico para diagnóstico pulpar. Paralelamente, realizava-se o exame radiográfico. Os dados clínicos nos possibilitaram estabelecer o grau e extensão da inflamação pulpar, e o exame radiográfico nos possiblitou comprovar ou detectar alterações periapicais em alguns casos de pulpites.

Um terceiro elemento ("exame clínico visual da polpa") , que descreveremos na técnica da pulpotomia, foi utilizado como "dado decisivo" na avaliação das condições da polpa, indicação da pulpotomia e prognóstico.

Para desinflamar a polpa radicular foram utilizadas as seguintes substâncias anti-inflamatórias associadas a antibiótico ou furacin: Rifocort (pomada): prednisolona mais antibiótico - Omcilon - $A-M$ (pomada): trancinolona mais antibiótico - Acetato de Prednisolona e Furacin Oto-solução em partes iguais.

Para recobrimento pulpar foi utilizado o hidróxido de cálcio pró-análise (quimicamente puro), manipulado com água destilada ou soro fisiológico.

No selamento da cavidade foi empregado um cimento à base de óxido de zinco e eugenol, pulpo-san.

\section{TÉCNICA DA PULPOTOMIA}

Estabelecido o-diagnóstico de pulpite, tinha início a pulpotomia obedecendo a seguinte sequência:

10 - Radiografia Prévia.

$2^{\circ}$ - Anestesia terminal ou regional.

$3^{\circ}$ - Sempre que possível isolamento absoluto do campo operatório.

40. - Abertura coronária pela técnica de Berk e Krakov ( 2 ), na qual 
- assoalho da cavidade estende-se $2 \mathrm{~mm}$ além do limite da câmara pulpar.

50 - Amputação da polpa coronária com escavador em forma de colher bem afiado.

60 - Irrigação da câmara pulpar com água destilada, ou soro fisiológico.

70. - Secagem e homostasia com bolas de algodão autoclavado.

8\% - "Exame clínico visual da polpa" para avaliar as condições do tecido pulpar. Esta é, talvez, a fase mais importante da pulpotomia e consiste em analisar e avaliar a hemorragia durante a amputação da porção coronária da polpa e, posteriormente, a consistência da polpa radicular.

A hemorragia deve ser, preferentemente, abundante com sangue de coloração vermelho vivo. Pouca hemorragia e sangue com coloração rósea e aspecto de aguado, indicam polpa severamente comprometida, e contra indicam a pulpotomia.

No que se refere à polpa radicular, esta deve apresentar as seguintes características: deve ter corpo, ser consistente, firme e ao mesmo tempo elástica, isto é, quando pressionada volta à posição anterior. A sua coloração deve ser vermelho vivo, Polpa radicular sem consistência, pastosa, isquêmica, ou semi liquefeita indica tecido severamente alterado e sem condições de conservação.

90 - Aplicação sobre a polpa radicular do medicamento à base de corticosteroide associado a antibiótico ou furacin por 48 horas.

$10^{\circ}$. - Colocação de uma bola de algodão autoclavado na câmara pulpar e selamento da cavidade com óxido de zinco e eugenol (pulpo-san). 11 ? - Após 48 horas, remoção do curativo e irrigação da câmara pulpar com água destilada, soro fisiológico ou água de cal. Secagem com algodão autoclavado.

$12^{\circ}$ - Aplicação do hidróxido de cálcio pró-analise, manipulado com água destilada, sobre a polpa radicular.

130 - Selamento hermético da cavidade com pulpo-san.

\section{CONTROLE PÓS OPERATÓRIO}

Na avaliação do quadro pós operatório foram considerados os seguintes aspectos:

a) - Quadro clínico: a ausência de sintomas é um bom sinal, todavia não é indicação absoluta de sucesso.

b) - Teste elétrico: o teste elétrico, na maioria das vezes, foi positivo. Ass vezes, negativo, devido à profundidade do remanescente pulpar. Nestes casos era removido o material de proteção pulpar e aplicado o pulpo teste diretamente na entrada dos canais.

c) - Exame clínico visual da barreira de dentina: A partir de sessenta dias em diante removia-se o selamento e o hidróxido de cálcio para constatar a presença da barreira dentinária. Tal procedimento era realizado visualmente ou através do tato por meio de instrumentos endodônticos.

d) - Exame radigráfico: era realizado a partir do segundo mês em diante para constatar:

10. - No local da amputação: formação da ponte de dentina.

20 - Na região apical: ausência, redução ou desaparecimento de área radiolúcida periapical, periápice normal, lâmina dura íntegra.

No pré operatório, o aspecto clínico visual da polpa foi classificado como "BOM, "REGULAR", "MAU".

"BOM", quando a hemorragia era abundante e com sangue de coloração 
vermelho vivo, e a polpa bem consistente, firme e de coloração vermelho vivo.

"REGULAR", quando a hemorragia era de média intensidade e sangue vermelho vivo, polpa não muito consistente e firme, porém de coloração vermelho vivo.

"MAU", quando a hemorragia era de pequena intensidade e com sangue não muito vermelho e a polpa com pouca consistência e firmeza e coloração tendendo para o rosa.

\section{RESULTADOS}

Os resultados obtidos podem ser classificados em imediatos e mediatos. $\mathrm{O}$ resultado imediato foi o alívio da dor em $100 \%$ dos casos. Os resultados mediatos, existosos após um período de proservação de até dezoito meses, foram:
10. - Permanência da polpa radicular viva e assintomática.

$2^{\circ}$ - Ausência, redução ou desaparecimento de área Radiolúcida periapical.

$3^{\circ}$ - Resposta normal aos testes mecânicos, termicos e elétricos.

40 - Periápice normal e lâmina dura íntegra.

50 - Formação de ponte de dentina, constatável por meios clínicos ou radiográficos.

O número total de dentes tratados foi 52, sendo 41 molares inferiores e 11 molares superiores. Deste total, 46 foram acompanhados por um período de proservação de 6 a 18 meses e, 6 foram acompanhados por um a dois meses. Dos 46 casos cuja proservação foi de 6 a dezoito meses, 3 apresentavam canal único, o que dá um total de 132 canais, nos quais o controle foi de 6 a 18 meses. Quadro 1 .

\section{QUADRO 1}

\begin{tabular}{|c|c|c|}
\hline DENTES & TR A T A D O S \\
\hline TOTAL $=52$ & $11=$ Molares inferiores \\
& $46=$ proservação de 6 a 18 meses \\
52 & $6=$ proservação de um a 2 meses \\
46 & 3 casos: canal único $=\quad$ total 132 canais.
\end{tabular}

No quadro 2 temos uma visão panorâmica dos 52 casos tratados, com os respectivos diagnósticos clínicos, aspecto clínico visual das polpas, medicamentos usados, número de sucessos e insucessos e percentual geral de êxito. 


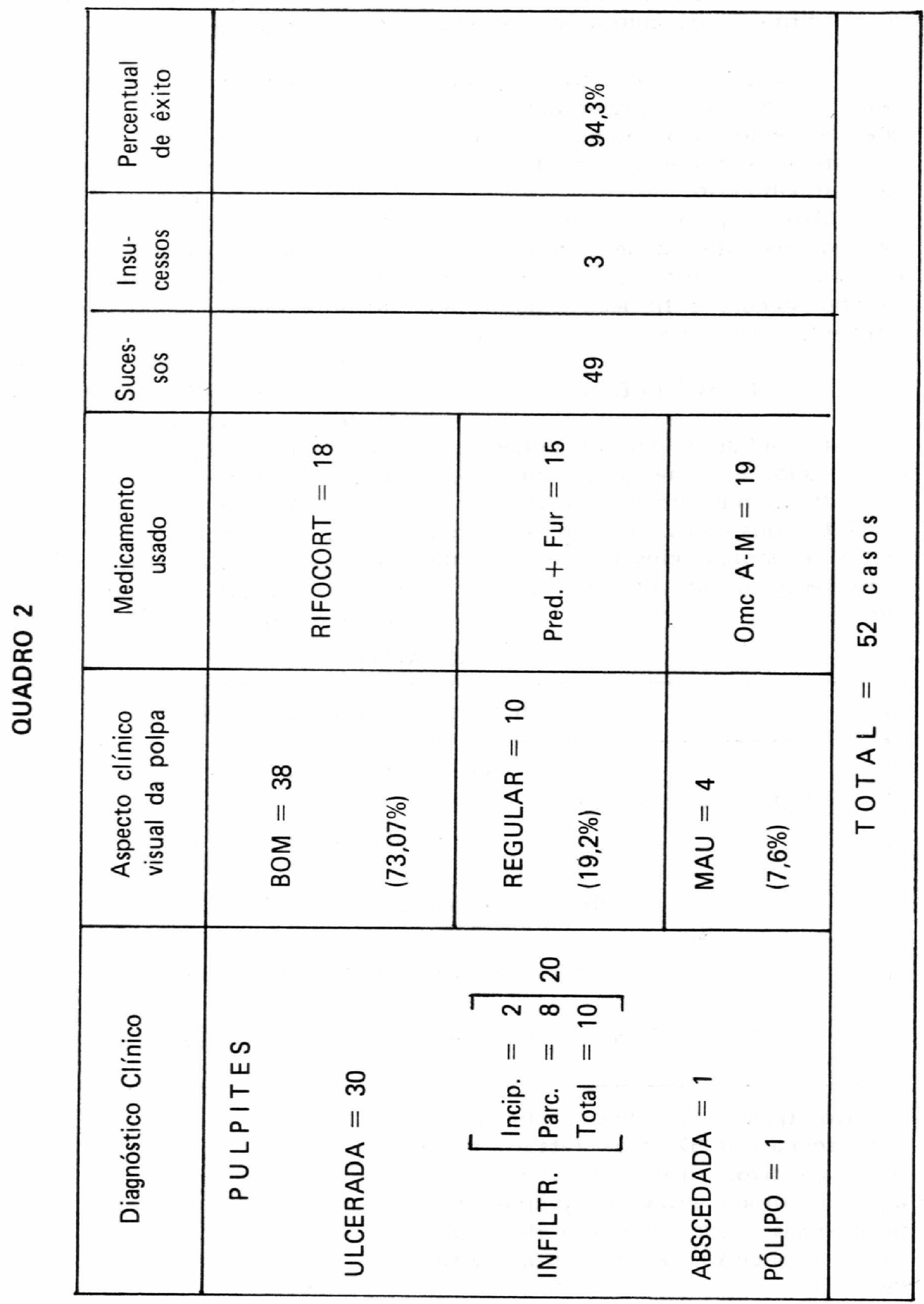


No quadro 3 podemos observar os trinta casos cujo diagnóstico clínico foi pulpite ulcerada, o aspecto clínico visual das polpas, os medicamentos empregados e os resultados. Dos $30 \mathrm{ca}$ sos tratados, 28 foram exitosos.
No quadro 4 temos os 20 casos cujo diagnóstico foi pulpite infiltrativa, o aspecto - clínico visual das polpas, os medicamentos empregados e os resultados. Dos 20 casos tratados, 19 foram exitosos e um mal sucedido.

QUADRO 3

\begin{tabular}{|c|c|c|c|c|}
\hline $\begin{array}{l}\text { Diagnóstico } \\
\text { Clínico }\end{array}$ & $\begin{array}{l}\text { A specto cl ínico } \\
\text { visual da polpa }\end{array}$ & $\begin{array}{l}\text { Medicamento } \\
\text { usado }\end{array}$ & $\begin{array}{l}\text { Suces- } \\
\text { sos }\end{array}$ & $\begin{array}{l}\text { Insu- } \\
\text { cessos }\end{array}$ \\
\hline \multirow{8}{*}{$\begin{array}{l}\text { Pulpite } \\
\text { Ulcerada }=30\end{array}$} & \multirow{3}{*}{ BOM $=20$} & Rifocort $=6$ & 5 & 1 \\
\hline & & Pred-Fur $=6$ & 6 & \\
\hline & & Omc $A-M=8$ & 8 & \\
\hline & \multirow[b]{2}{*}{ REGULAR $=7$} & Rifocort $=1$ & 1 & \\
\hline & & Pred-Fur $=4$ & 3 & 1 \\
\hline & \multirow{3}{*}{$M A U=3$} & $O m c A-M=2$ & 2 & \\
\hline & & Rifocort $=1$ & 1 & \\
\hline & & $O m c A-M=2$ & 2 & \\
\hline
\end{tabular}

TOTAL $=30$ casos

QUADRO 4

\begin{tabular}{|c|c|c|c|c|}
\hline $\begin{array}{l}\text { Diagnóstico } \\
\text { clínico }\end{array}$ & $\begin{array}{l}\text { Aspecto clínico } \\
\text { visual da polpa }\end{array}$ & $\begin{array}{l}\text { Medicamento } \\
\text { usado }\end{array}$ & $\begin{array}{l}\text { Suces- } \\
\text { sos }\end{array}$ & $\begin{array}{l}\text { Insu- } \\
\text { cessos }\end{array}$ \\
\hline \multirow{2}{*}{$\begin{array}{l}\text { Pulpite } \\
\text { Infiltrativa } \\
\text { Incipiente } \\
=2\end{array}$} & $\mathrm{BOM}=1$ & Pred-Fur $=1$ & 1 & \\
\hline & REGULAR $=1$ & Rifocort $=1$ & 1 & \\
\hline \multirow{4}{*}{$\begin{array}{l}\text { Pulpite } \\
\text { Infiltrativa } \\
\text { Parcial } \\
=8\end{array}$} & \multirow{3}{*}{ BOM $=7$} & Rifocort $=2$ & 2 & 1 \\
\hline & & Pred-Fur $=2$ & 2 & \\
\hline & & Omc. $A-M=2$ & 2 & \\
\hline & REGULAR $=1$ & Pred-Fur $=1$ & 1 & \\
\hline \multirow{4}{*}{$\begin{array}{l}\text { Pulpite } \\
\text { Infiltrativa } \\
\text { Total } \\
=10\end{array}$} & \multirow{3}{*}{$\mathrm{BOM}=9$} & Rifocort $=5$ & 5 & \\
\hline & & Pred-Fur $=1$ & 1 & \\
\hline & & Omc. $A-M=3$ & 3 & \\
\hline & REGULAR $=1$ & Omc. $A-M=1$ & 1 & \\
\hline
\end{tabular}


No quadro 5 temos o caso de pulNo quadro 6 temos o caso do pópite abscedada, o aspecto clínico visual lipo pulpar, o aspecto clínico visual da polpa, o medicamento empregado e da polpa, o medicamento empregao resultado, que foi exitoso. do e o resultado, que foi exitoso.

QUADRO 5

\begin{tabular}{|l|c|c|c|c|}
\hline \multicolumn{1}{|c|}{$\begin{array}{c}\text { Diagnóstico } \\
\text { clínico }\end{array}$} & $\begin{array}{c}\text { Aspecto cl ínico } \\
\text { visual da polpa }\end{array}$ & $\begin{array}{c}\text { Medicamento } \\
\text { usado }\end{array}$ & $\begin{array}{c}\text { Suces- } \\
\text { so }\end{array}$ & $\begin{array}{c}\text { Insu- } \\
\text { cesso }\end{array}$ \\
\hline $\begin{array}{l}\text { PULPITE } \\
\text { ABSCEDADA }=1 \\
\text { (presença de pús } \\
\text { na câmara pulpar) }\end{array}$ & REGULAR $=1$ & RICOFORT =1 & 1 & \\
\hline
\end{tabular}

QUADRO 6

\begin{tabular}{|c|c|c|c|c|}
\hline $\begin{array}{c}\text { Diagnóstico } \\
\text { clínico }\end{array}$ & $\begin{array}{c}\text { A specto clínico } \\
\text { visual da polpa }\end{array}$ & $\begin{array}{c}\text { Medicamento } \\
\text { usado }\end{array}$ & $\begin{array}{c}\text { Suces- } \\
\text { usado }\end{array}$ & $\begin{array}{c}\text { Insu- } \\
\text { cesso }\end{array}$ \\
\hline PÓLIPO $=1$ & MAU $=1$ & Omc. A-M -1 & 1 & \\
\hline
\end{tabular}

No quadro 7 temos os três casos de insucesso, onde estão relacionados os diagnósticos clínicos, o aspecto clínico visual das polpas, os medicamentos usados e as causas prováveis de insucesso.

\begin{tabular}{|c|c|c|c|}
\hline & CASOS DE & S U ESSO & \\
\hline $\begin{array}{l}\text { Diagnóstico } \\
\text { clínico }\end{array}$ & $\begin{array}{l}\text { Aspecto dínico } \\
\text { visual da polpa }\end{array}$ & $\begin{array}{l}\text { Medicamento } \\
\text { usado }\end{array}$ & $\begin{array}{l}\text { Causas prováveis } \\
\text { do insucesso }\end{array}$ \\
\hline \multirow[t]{2}{*}{$\begin{array}{l}\text { PULPITE } \\
\text { ULCERADA }=2\end{array}$} & $\mathrm{BOM}=1$ & Ricofort & $\begin{array}{l}\text { Queda do sela- } \\
\text { mento da cavi- } \\
\text { dade. }\end{array}$ \\
\hline & REGULAR $=1$ & Pred-Fur. & $\begin{array}{l}\text { Consistência regu- } \\
\text { lar e provável } \\
\text { presen ça de coá- } \\
\text { gulo extra pulpar }\end{array}$ \\
\hline $\begin{array}{l}\text { PULPITE } \\
\text { Infiltr. } \\
\text { PARCIAL = } 1\end{array}$ & $\mathrm{BOM}=1$ & Ricofort & $\begin{array}{l}\text { Usou medicação } \\
\text { prévia na cavidade } \\
\text { para alívio da dor. } \\
\text { Provável agressão } \\
\text { química da polpa. }\end{array}$ \\
\hline
\end{tabular}


No quadro 8 observamos o seguinte: das 132 polpas inflamadas (46 molares, 3 com canal único) submetidas à pulpotomia, curativo com corticosteroide-antibiótico ou corticosteroide-furacin. e, após aplicação de hidróxido de cálcio, 127 permaneceram vivas, assintomáticas e com respostas normais aos testes mecânicos, térmicos e elétricos e périapice normal, ou seja, em $96,2 \%$ a pulpite foi reversivel.

Das 127 polpas que permaneceram vivas, assintomáticas e com resposta normal aos testes mecânicos, térmicos e elétricos e périápice normal, 112 formaram barreira de dentina, isto é, $88,1 \%$, e apenas cinco polpas necrosaram, ou seja, "em somente 3,7\% a pulpite foi irreversivel", quadro 8.

Um fato curioso que chamou a atenção é que em nunhum caso, dos 112 com barreira de dentina, a ponte de dentina foi visível na radiografia. Isto, segundo Holland (12 14 - 15 ), deve-se à pouca espessura ou pouca radiopacidade da barreira dentinária.

Dos 52 dentes tratados, 22 evidenciaram lesão apical (espessamente ou rarefação), ou seja, a média de incidência de lesões apicais encontrada em casos de pulpite, foi de $42,3 \%$, assim distribuidas - Quadro 9:

QUADRO 8

\begin{tabular}{|c|c|c|c|}
\hline \multicolumn{4}{|c|}{ CASOS ACOMPANHADOS POR 6 A 18 MESES } \\
\hline Número de casos & $\begin{array}{l}\text { Polpa viva e } \\
\text { assintomática }\end{array}$ & $\begin{array}{c}\text { Barreira } \\
\text { dentinária }\end{array}$ & Necrose pulpar \\
\hline $\begin{array}{c}46 \text { MOLARES } \\
\text { ( } 3 \text { com canal único }) \\
\text { Total }=132 \text { polpas. } \\
(100 \%)\end{array}$ & $\begin{array}{c}127 \\
(96,2 \%) \\
\text { REVERSIVEL }\end{array}$ & $\begin{array}{c}112 \\
(88,1 \%)\end{array}$ & $\begin{array}{c}5 \\
(3,7 \%) \\
\text { IRREVERSIVEL }\end{array}$ \\
\hline
\end{tabular}

QUADRO 9

\begin{tabular}{|c|c|c|}
\hline \multicolumn{2}{|c|}{ Diagnóstico Clínico } & \multirow{2}{*}{$\begin{array}{l}\text { Diagnóstico Radiográfico: } \\
\text { No de lesões apicais } \\
12\end{array}$} \\
\hline Pulpite Ulcerada & $=30$ & \\
\hline Pulpite Infiltr. Parc. & $=8$ & 3 \\
\hline Pulpite Infiltr. Total & $=10$ & 5 \\
\hline Pulpite Abacedada & $=1$ & 1 \\
\hline Pólipo Pulpar & $=1$ & 1 \\
\hline
\end{tabular}


É interessante notar que em 8 casos de diagnóstico clínico de pulpite infiltrativa parcial (inflamação apenas da polpa coronária), 3 apresentaram lesão apical constatável radiograficamente - Quadro 9.

Dos 22 dentes molares que apresentaram lesão apical, um tinha canal único, o que dá um total de 64 polpas. Destas 64 polpas inflamadas e com lesão apical concomitante submetidas à pulpotomia, curativo com corticosteroide-antibiótico ou corticosteroidefuracin e, após aplicação de hidróxido de cálcio, 64 permaneceram vivas e assintomáticas, com respostas normais aos testes mecânicos, térmicos e elétricos, ou seja, em $100 \%$ dos casos a pulpite foi reversível.

Também em todos os casos (100\%) houve regressão e reparação da lesão apical e, em 60 polpas formou-se ponte de dentina, isto é, em $93,7 \%$ - quadro 10 .

QUADRO 10

\begin{tabular}{|c|c|c|c|c|}
\hline \multicolumn{5}{|c|}{ Lesões apicais: Espessamento ou Rarefação } \\
\hline $\begin{array}{c}\text { Número } \\
\text { de dentes }\end{array}$ & $\begin{array}{c}\text { Número } \\
\text { de canais }\end{array}$ & Polpa Viva & $\begin{array}{c}\text { Repressão da } \\
\text { lesão apical }\end{array}$ & $\begin{array}{c}\text { Barreira de } \\
\text { de dentina }\end{array}$ \\
\hline $\begin{array}{c}22 \\
\text { (1 com canal } \\
\text { único })\end{array}$ & $\begin{array}{c}64 \\
(100 \%)\end{array}$ & $\begin{array}{c}64 \\
(100 \%)\end{array}$ & $\begin{array}{c}64 \\
100 \%)\end{array}$ & $\begin{array}{c}60 \\
(93,7 \%)\end{array}$ \\
\hline Irreversível & Irreversível & & \\
\hline
\end{tabular}

$\mathrm{Na}$ fotografia 1 podemos observar a radiografia prévia de um dente com pulpite ulcerada com espessamento do pericemento apical. Na fotografia 2 , um ano após, periápice normal com lâmina dura íntegra. 

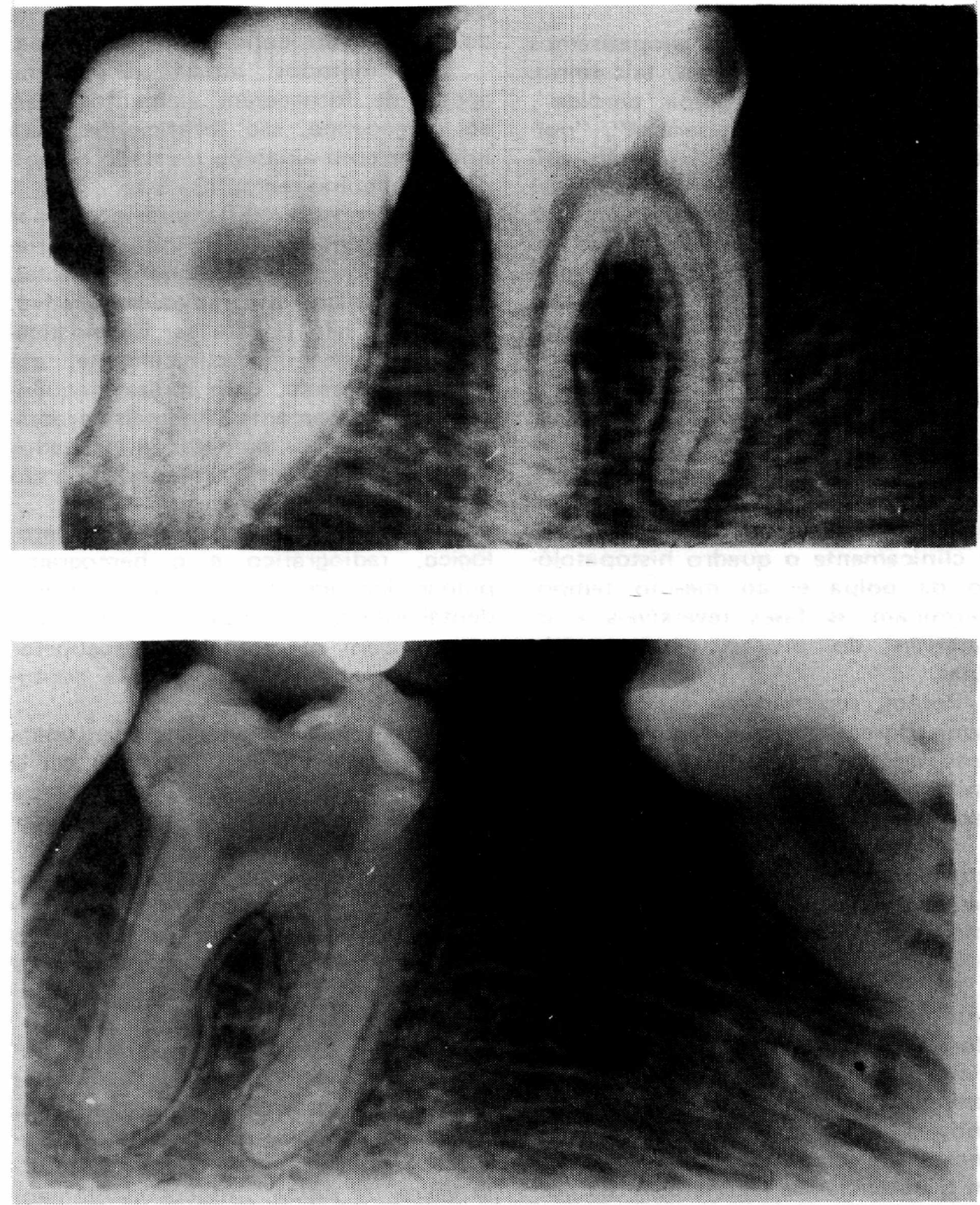

R.Fac.Odont., Porto Alegre, 27:153-171, 1985. 


\section{DISCUSSÃo}

Em verdade, nunca simpatisamos com afirmativas categóricas, tais como: "polpa inflamada é polpa perdida", "polpa exposta é irrecuperável", "pulpite é lesão irreversível", "lesão apical é sempre sequela de necrose total da polpa ou de involvimento pulpar irreversível", etc., e tantas outras semelhantes, dando a enteder que toda tentativa de conservação ou de tratamento pulpar seria em vão e de êxito duvidoso ou impossível.

Inúmeros autores $(6-10-19$ $25-30-33$ ), associam o termo "pulpite" com lesão irreversível e, consequentemente, prognóstico desfavorável.

Como vimos na revisão da literatura, há autores que pretendem estabelecer clinicamente o quadro histopatológico da polpa e, ao mesmo tempo, determinam as fases reversíveis $\mathrm{e}$ irreversíveis do processo inflamatório pulpar.

Porém, um número espressivo de pesquisadoree comprovou experimentalmente a falta de correlação entre as manifestações clínicas e o quadro histopatológico da polpa.

Ainda outros, mencionam a simultaneidade da lesão pulpar e periapical, e alguns consideram esta situação como definitivamente irreversível para a polpa.

O diagnóstico pulpar é absolutamente essencial no tratamento conservador. O diagnóstico da inflamação e da extensão da inflamação pulpar é relativamente fácil de ser estabelecido. A grande dificuldade é, realmente, estabelecer até quando podemos contar com o potencial de recuperação. da polpa, isto é, até que ponto o processo inflamatório pulpar é reversível ou irreversível (5).
Em torno disso é que se situa grande parte da controvérsia e da polêmica do tratamento conservador da polpa.

Os métodos atuais de exâme apesar de fornecerem elementos úteis ao diagnóstico, são insuficientes para informar com exatidão o estado da polpa dental como tecido (3 - 5).

O processo inflamatório pulpar apresenta uma gama infinita de variações, como: incipiente, parcial, total, agudo, crônico, infiltrativo, supurativo, etc. , que não podem ser diagnosticadas com exatidão clinicamente, até porque, ocorrem, com muita frequência, simultaneamente. Ademais, processos degenerativos pulpares já instalados (degeneração fibrosa, reticular), não são identificados clinicamente.

O diagnóstico clínico, histopatológico, radiográfico e o hemograma pulpar indicam apenas que a polpa dental está doente mas, absolutamente, não revelam o potencial de recuperação desta polpa doente e, não permitem estabelecer o prognóstico.

Nos parece óbvio que, a única maneira de avaliarmos até onde vai o potencial de recuperação da polpa dental inflamada, é a de submeter a tratamento conservador (pulpotomia) todas as formas de pulpites identificadas pelo diagnóstico clínico, radiográfico e histopatológico (neste último caso admitindo a correlação entre os diagnósticos clínico e histopatológico) e analisar os resultados.

Este foi um dos objetivos deste trabalho. Todavia, para estabelecermos as condições da polpa e o prognóstico, usamos como dado ou elemento de avaliação, exclusivamente, o"aspecto clínico visual da polpa", independentemente de qualquer outra manifestação clínica, radiográfica ou do possível quadro histopatológico presente. 
Embora a polpa esteja totalmente inflamada, a ponto de provocar lesões apicais, ela é tratável. Consegue-se a preservação do tecido pulpar e a volta dos tecidos periapicais à normalidade, - que foi constatado clínica, radiográfica e histológicamente (15). Fica evidente, portanto, que a extensão do processo inflamatório pulpar (incipiente, parcial, total) não constitui elemento de contra indicação do tratamento conservador, e em decorrência, também, parece não haver mérito maior em se pretender determinar clinicamente se o processo inflamatório é incipiente, parcial ou total.

Pela combinação de um acurado diagnóstico, com uma adequada seleção do caso e uma correta técnica de tratamento, o sucesso é prognosticável e é, quase sempre, a regra.

Uma polpa que apresente características compat1veis com as de um tecido vivo é o maior pré-requisito para o sucesso.

De outra parte, a ação do corticosteroide associado ao antibiótico ou furacin, como anti-flogístico ou controlador do processo inflamatório pulpar, parece ser pacífica e se constitui num elemento indispensável no tratamento conservador da polpa inflamada.

Finalmente, tendo em vista fatores econômicos, técnicos e inexistência de tratamento endodôntico em serviços assistenciais e previdenciários, devemos optar por uma terapêutica eficiente, mais simples, mais rápida e menos dispendiosa como a pulpotomia, sobretudo considerando que, nestas circunstâncias, se esta terapêutica não for preconizada, a outra alternativa de tratamento será a exodontia com a consequente mutilação. de inúmeros pacientes.
Ademais, os eventuais insucessos da pulpotomia não são insucessos definitivos, pois sempre existe a possibilidade de tratamento endodôntico.

\section{CONCLUSÕES}

De acordo com a literatura con-sultada e segundo a evidência clínica dos nossos resultados, chegamos às seguintes conclusões:

10 - O diagnóstico clínico radiográfico não revela as reais e exatas condições do tecido pulpar (inflamação aguda, crônica, incipiente, parcial, total, infiltrativa, abscesso, degeneração, etc.) e não tem relação com o prognóstico.

20 - O diagnóstico clínico radiográfico não permite estabelecer lesões pulpares irreversíveis.

30. - O "exame clínico visual da polpa e as características do sangue pulpar" constituem-se em elementos de real valor na determinação do estado da polpa e do prognóstico. Isto é, na determinação da reversibilidade ou irreversibilidade da lesão pulpar, ou ainda, do potencial de recuperação da polpa, independentemente do quadro histopatológico e de qualquer outra manifestação clínica ou radiográfica. Sua adoção como elemento auxiliar de diagnóstico e prognóstico permite atingir a expressiva cifra de cerca de $95 \%$ de êxito no tratamento conservador da polpa dental inflamada. 40. - A polpa dental clinicamente inflamada, dependendo do seu aspecto clínico visual, pode ser submetida a tratamento conservador pela pulpotomia com cerca de $95 \%$ de êxito.

50. - O tratamento conservador pela pulpotomia está indicado em polpas inflamadas, pois mesmo nos casos sem formação de ponte de den- 
tina, a polpa mantem-se viva, assintomática, com respostas normais aos testes mecânicos, térmicos e elétricos, e estruturas periapicais e lâmina dura normais.

60 - Os corticosteroides associados à pulpotomia mostraram-se eficazes no controle da dor em $100 \%$ dos casos.

70 - A nível clínico, a associação corticosteroide-antibiótico ou coricosteroide-furacin, nas concentrações e tempos utilizadós sobre a polpa, não só não retarda como favorece o processo de reparação e cicatrização pulpar.

$8^{\circ}$ - $O$ tempo ideal de permanência do corticoide em contato com a polpa é de 48 horas. Porém, nos casos em que este tempo foi prolongado por três ou cinco dias, não foram observadas alterações na evolução e nos resultados dos tratamentos.

90. - Em nenhum caso foram observados efeitos sistêmicos colaterais resultantes do uso de corticosteroides.

$10^{\circ}$ - A maior dificuldade no tratamento conservador da polpa dental inflamada consiste em determinar ou avaliar o potencial de recuperação da polpa apenas pelos sintomas clínicos. Estes, na maioria das vezes, são insuficientes e conduzem a diagnóstico e prognóstico imprecisos.

110 - O método clínico é superior ao radiográfico para avaliação da presença e da extensão da ponte de dentina.

$12^{\circ}$ - Mesmo que a ponte de dentina não seja formada, se a polpa permanece viva, assintomática, com respostas normais aos testes mecânicos, térmicos e elétricos e extruturas periapicais normais, a pulpotomia é considerada exitosa.
13ㅇ - As falhas na formação da barreira de dentina foram observadas com mais frequência em polpa de pequeno volume.

14ㅇ - Dor espontânea, intensa, contínua, sensibilidade à percussão, espessamento ou rarefação apical, não são indicativos de lesão pulpar irreversível e não constituem contra indicação para a pulpotomia, desde que o "aspecto clínico visual da polpa" seja bom.

150 - Não há relação entre dor espontânea, intensidade e duração da dor com extensão da inflamação pulpar.

160 - A ponte dentinária, por si só, não serve como critério clínico de cicatrização pulpar. A observação da barreira dentinária em dente com polpa necrosada, mostra que a barreira dentinária pode ocorrer em polpas com inflamação irrevesível.

170 - Lesões pulpares diagnosticadas clinicamente como pulpite infiltrativa parcial, pulpite inflitrativa total, pulpite abscedada, pulpite ulcerada e- pólipo, com ou sem envolvimento periapical, são reversíveis desde que "o aspecto clínico visual da polpa" seja bom, e quando submentidas à pulpotomia, ação de corticoideantibiótico ou corticoide-furacin por 48 horas e, após hifróxido de cálcio. 18 ? - No período de proservação de até dezoito meses não foi constatado nenhum caso de reabsorção dentinária interna.

190 - Reabsorção dentinária interna indica a presença de pulpite granulomatosa, e deve ser considerada como insucesso.

20․ - Exames histológicos mostram que, na maioria dos casos, a inflamação localiza-se na polpa coronária, permanecendo a polpa radicular normal ou pouco comprometida. 
21으 - A incidência de lesão periapical simultaneamente com a inflamação da polpa é da ordem de $42,3 \%$. 22 ? - O desaparecimento da rarefação periapical (constatada em $100 \%$ dos casos) após o tratamento conservador, é mais significativo do que a presença da barreira de dentina e sugere a cura da pulpite.

$23^{\circ}$ - $\mathrm{O}$ alto índice de sucesso da pulpotomia $(94,3 \%)$, mostra de forma definitiva o pouco mérito e ineficácia do relacionamento do quadro clínico com o histopatológico e deste com o prognóstico, já que, praticamente, todas as variantes do processo inflamatório pulpar, diagnosticadas clinicamente, constituem-se em lesões reversíveis, desde que submetidas a tratamento adequado após rigoroso "exame clínico visual da polpa". 240 - Clinicamente é impossível estabelecer com exatidão, através dos sintomas e pelos testes macânicos, térmicos e elétricos, se o quadro histopatológico pulpar é infiltrativo parcial, infiltrativo total, abscedado, e se é agudo ou crônico. Mas, ainda que isto fosse possível, nós estaríamos estabelecendo apenas o quadro histopatológico e não avaliando o potencial de recuperação da polpa. Portanto, a coincidência do diagnóstico clínico com o histopatọlógico seria apenas uma constatação de laboratório, mas que não avalia o potencial de recuperação da polpa e consequentemente não revela e nem permite prever se a lesão pulpar é reversível ou irreversível, daí o pouco mérito em se pretender determinar o quadro histopatológico pulpar.

250 - A reversíbilidade ou irreversibilidade das pulpites só pode ser determinada submetendo a tratamento adequado todas as variantes do pro- cesso inflamatório pulpar (pulpites infiltrativa incipientes, parcial, total, abscedada, ulcerada e pólipo) e analisando os resultados. No presente trabalho, tratadas todas as variantes do processo inflamatório pulpar, encontramos um percentual de reversibilidade de $94,3 \%$, conforme quadros demostrativos.

26․ - Em face deste percentual de êxito, a questão da correlação entre - diagnóstico clínico e o histopatológico, fica restrita a duas alternativas: a) - Ou "o diagnóstico clínico não coincide com o histopatológico", e neste caso as polpas consideradas, clinicamente, como severamente inflamadas, em realidade não o são. São lesões apenas incipientes, reversíveis e consequentemente tratáveis.

b) - Ou "o diagnóstico clínico coincide com o histopatológico", e neste caso temos que admitir que as polpas, mesmo severamente inflamadas, possuem elevados potencial de recuperação, isto é, são, na sua grande maioria, lesões reversíveis e portanto tratáveis, conforme quadros demonstrativos.

27ํ - Não há correlação entre diagnóstico clínico e o "aspecto visual da polpa".

$28^{\circ}$ - Os resultados obtidos e a evidência clínica nos autorizam a afirmar que "o exame clínico visual da polpa", permite prever ou avaliar o potencial de recuperação pulpar em até cerca de $95 \%$ dos casos, submetidos à pulpotomia, aplicação de corticosteroide-antibiótico ou corticosteroide-furacin por 48 horas, e posteriormente, hidróxido de cálcio, qualquer que seja o quadro clínico, histopatológico, ou radiográfico.

290 - A indicação final da pulpotomia não é baseada no diagnóstico clínico, nem no possível quadro histo- 
patológico e nem no diagnóstico radiográfico, mas sim no “exame visual da polpa" e nas "características e intensidade da hemorragia pulpar".

$30^{\circ}$. - Finalmente, 0 conceito de que lesão periapical é sempre sequela de necrose total da polpa ou envolvimento pulpar irreversível, deve ser reformulado.

310 - Podemos enumerar como causas de insucesso da pulpotomia, as seguintes:

a) - Diagnóstico incorreto da condição pulpar.

b) - Presença de fragmentos de dentina sobre a ferida pulpar.

c) - Presença de coágulo extra pulpar.

d) - Dilaceração do tecido pulpar durante a secção da polpa coronária. e) - Infiltração marginal.

f) - Ação nociva e irritante dos medicamentos usados.

Estes fatores são a causa do grande desacordo na porcentagem de sucesso da pulpotomia que, segundo a literatura, varia de $30 \%$ a quase $100 \%$.

\section{SUMMARY}

The author evaluate clinically and radiographically the behaviour of the inflamed dental pulp, subjected to pulpotomy, corticoid-antibiotic action and after calcium hydroxide. Like diagnostic and prognostic data it is, exclusively considered the "clinic visual aspect of the pulp". The success was $94,3 \%$.

\section{REFERÊNCIAS BIBLIOGRAFICAS}

1. AlVARES, S. \& PAIVA, J.G. Variação da dor durante a evolução do processo inflamatório da polpa. Ars Curandi Odontol. , 1(3): 3-5, ago./set. 1974.

2. BERK, H. \& KRAKOW, A.A. Efficient Vital Pulp therapy. Dent. Clin. North Am. : :373-85, Jul. 1965.

3. BEVIÇACQUA. S. A fórmula leucocitária local nas inflamações pulpares. Rio de Janeiro, 1957. p.6 Tese.

4. - p.43.

5. - p.73.

6. DE DEUS, Q.D. Endodontia. 2.ed. Rio de Janeiro, Guanabara Koogan, 1976. p. 183.

7. FILGUEIRAS, J. et alli. Edodontia clínica. Rio de Janeiro, Científica, 1962. p.212.

8. - p.223.

9. FOREMAN. P.C. Resolution of periapical radiolucency following renewal of the pulpotomy dressing. Int. Dent. J. , 13(1): 41-3, Jan. 1980.

10. GROSSMAN, L.I. Tratamento dos canais radiculares. Trad. Sylvio Bevilacqua. Rio de Janeiro, Atheneu, 1954. p.79-80.

11. HOLLAND, R. \& SOUZA, V.O. problema do diagnóstico clínico e indicação do tratamento da polpa dental inflamada. Rev. Assoc. Paul. Cir. Dent. , 24(5): 188-93, set./out. 1970. 
12. HOLLAND, R. et alii. Edodontia. Araçatuba, s.ed. 1972. p.116.

13. HOLLAND, R. \& SOUZA, V. Treatment of the inflamed dental pulp. Aust. Dent. J., 19(3): 191-6, Jun. 1974.

14. - Tratamento conservador da polpa dental. Ars. Curandi Odontol. , 2(2): 3-17, Jun./Jul. 1975.

15. HOLLAND, R. \& SOUZA, V. Manual do curso intensivo de edodontia. 1976.

16. LANGLAND, K. et alii. Corticosteroids in dentistry. Int. Dent. J. , 27(3): 217-51, sept. 1977.

17. LASALA, A. Edodontia. 2,ed, CAracas, Cromotip, 1971. p.73.

18. MAISTO, O.A. Edodontia. Buenos Aires, Mundi, 1967. p.35.

19. .p.42.

20. .p. 126.

21. MILANO, N.F. Estudo da correlação entre os diagnósticos clínicos e histopatológicos. Porto Alegre, 1957. 127p. Tese.

22. MJOR. I. \& TRONSTAND, L. Experimentally induced pulpitis. Ora/ Surg., 34 (1): 102-8, Jul. 1972.

23. MOORE, D.L. Conservative treatment of teeth with vital pulps and periapical lesions: a preliminary report. J. Prosthet. Dent. , 18: 476-81, Nov. 1967.

24. PAIVA, J.G. \& ALVARES, S. Edodontia. São Paulo, Atheneu, 1979. p.1.

25. p.30.

26. p.96.

27. RUSSO, M.C. et alii. Treatment of inflamed pulp in decidious teeth. Histologieal study in dog. Bull. Tokyo. Dent. Coll. , 13(1): 9-20, Feb. 1972.

28. SCHROEDER, A. The aplication of corticosteroids in endodontics. D. practitioner \& D. Record, 13(10): 420-6, 1963.

29. SELTZER, S. et alii. The dynamics of pulp inflammation: correlation between diagnostic data and actual histologic findings in the pulp. Oral Surg. , 16: 856-71, 1963.

30. SELTZER, S. \& BENDER, I.B. A polpa dental. Rio de Janeiro, Labor, 1979. p.452.

31. SOUZA, V. \& HOLLAND, R. Treatment of the inflamed dental pulp. Aust. Dent. J. , 19(3): 191-6, 1974.

32. THOMA, K.H. A comparison of clinical roentgen and microscopical findings in 15 cases of infected pulps. J. Dent. Res. , 9:447. 1929.

33. VARELLA, J.A.F. \& PAIVA, J.G. Manual de edodontia. São Paulo, Isbra, 1963. p.47-8, 66.

34. VIA, W. Evaluation of deciduous molars treated by pulpotomy, and calcium hydroxide. J. Am. Dent. Assoc. , 50: 34-43, 1965. 